\title{
Constraining the pomeron structure using LHC data
}

\author{
Matthias Saimpert ${ }^{* \dagger}$ \\ CEA Saclay - Irfu/SPP \\ E-mail: matthias.saimpert@cea.fr
}

\section{Cyrille Marquet}

Centre de physique théorique, École Polytechnique, CNRS, 91128 Palaiseau, France

E-mail: cyrille.marquetecern.ch

\section{Christophe Royon}

CEA Saclay - Irfu/SPP

E-mail: christophe.royon@cea.fr

\section{Dominik Werder}

Department of Physics and Astronomy, Uppsala University, Box 516, SE-751 20 Uppsala,

Sweden

E-mail: dominik.werder@physics.uu.se

\begin{abstract}
We present here potential measurements at the LHC that can constrain the Pomeron, a colorless object which is exchanged during hard diffractive events in proton-proton collisions. So far, the Pomeron structure in terms of quarks and gluons has been extracted from QCD fits using HERA and Tevatron data. The LHC allows us to probe its structure in a completely new kinematical domain. The measurements which are discussed in this report use double Pomeron exchange (DPE) dijet and photon-jet final state processes, in which both protons escape the collision intact. Proton tagging is assumed and event generation is performed using the Forward Physics Monte Carlo (FPMC), a generator that has been designed to study forward physics, especially at the LHC.
\end{abstract}

International Conference on the Structure and the Interactions of the Photon including the 20th International Workshop on Photon-Photon Collisions and the International Workshop on High Energy Photon Linear Colliders

20 - 24 May 2013

Paris, France

\footnotetext{
* Speaker.

† We thank G. Ingelman and R. Peschanski for discussions and comments.
} 


\section{Dijet production in double Pomeron exchanges processes and gluon Pomeron structure}

The LHC provides high energy data at high luminosity in a completely new kinematical domain, which gives a unique opportunity to test further QCD [1]. One can first probe if the Pomeron observed at HERA ( $e p$ collider) is the same object than the one at the LHC ( $p p$ collider), that to say if we are sensitive to the same object. Forward proton tagging at the LHC [2] will allow the QCD evolution of the gluon and quark densities in the Pomeron to be tested and compared with the HERA measurements [3]. In particular, DPE production of dijet and photon-jet, which leave both protons intact, are especially sensitive respectively to the gluon and to the quark content of the Pomeron. The leading-order diagrams of those processes are shown in Fig. 1.
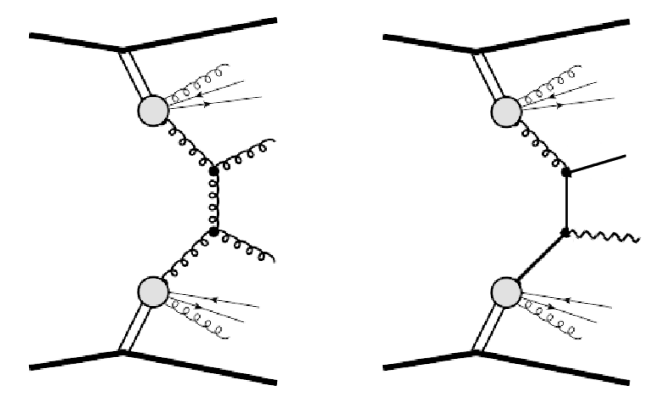

Figure 1: Leading-order diagrams for DPE dijet (left) and $\gamma+$ jet (right) production in proton-proton collisions. The dijet process is sensitive to the Pomeron gluon density and the $\gamma+$ jet process to the Pomeron quark densities.

The dijet production in DPE events at the LHC is sensitive to the gluon density in the Pomeron. In order to quantify how well we are sensitive to the gluon Pomeron structure, we display the differential dijet cross section as a function of the jet $p_{T}$ for proton-proton collisions at $\sqrt{s}=14 \mathrm{TeV}$ in Fig. 2 (top). The central black line shows the differential cross-section for the Pomeron gluon density measured at HERA, including an additional survival probability of 0.03 . The yellow band shows the effect of the $20 \%$ uncertainty on the gluon density taking into account the normalisation uncertainties. The dashed curves display how the dijet cross section at the LHC is sensitive to the gluon density distribution especially at high $\beta, \beta$ being the momentum fraction of the interacting parton coming from the Pomeron. For this sake, we multiply the gluon density of the Pomeron from HERA by $(1-\beta)^{v}$ where $v$ varies between -1 and 1 . When $v$ is equal to -1 (resp. 1), the gluon density is enhanced (resp, decreased) at high $\beta$. Fig. 2 shows that we can definitely check if the Pomeron model from HERA and its structure in terms of gluons is compatible between HERA and the LHC using dijet DPE events. This will be an important test of the Pomeron universality. This measurement can be performed for a luminosity as low as $10 \mathrm{pb}^{-1}$ since the cross section is very large (typically, one day at low luminosity without pile up at the LHC). It is worth noticing that this measurement will be limited by systematic uncertainties and not the statistical ones. Typically, if the jet energy scale is known with a precision of $1 \%$, we expect the systematics on the jet cross section mainly due to jet energy scale and jet $p_{T}$ resolution to be of the order of $15 \%$. However, from this measurement alone, it will be difficult to know if the potential difference between the 
expectations from HERA and the measurement at the LHC are mainly due to the gluon density or the survival probability since the ratios between the curves (varying the $v$ parameters) are almost constant.

An additional observable more sensitive to the Pomeron gluon density is displayed in Fig. 2 (bottom). This is the so-called dijet mass fraction, the ratio of the dijet mass to the total diffractive mass computed as $\sqrt{\xi_{1} \xi_{2} s}$ where $\xi_{1,2}$ are the proton fractional momentum carried by each Pomeron and $\sqrt{s}$ the center-of-mass energy of $14 \mathrm{TeV}$. We note that the curves corresponding to the different values of $v$ are much more spaced at high values of the dijet mass fraction, meaning that this observable is more sensitive to the gluon density at high $\beta$, as expected. This is due to the fact that the dijet mass fraction is equal to $\sqrt{\beta_{1} \beta_{2}}$. The measurement of the dijet cross section as a function of the dijet mass fraction is thus sensitive to the product of the gluon distribution taken at $\beta_{1}$ and $\beta_{2}$. It is worth mentioning that exclusive dijet events will contribute to this distribution at higher values of the dijet mass fraction $(>0.6-0.7)$ [4].
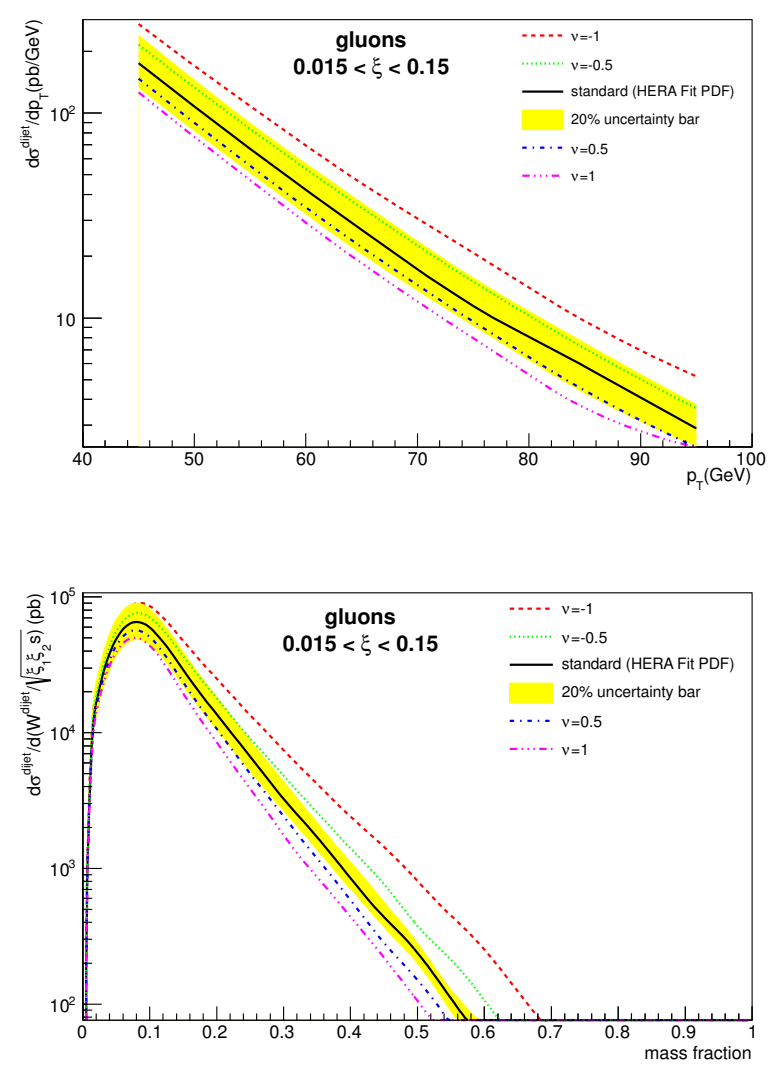

Figure 2: DPE dijet cross section as a function of the leading jet $p_{T}$ (top) and of the DPE dijet mass fraction distribution (bottom). The different curves correspond to different modifications of the Pomeron gluon density extracted from HERA data (see text). Proton-proton collisions at $\sqrt{s}=14 \mathrm{TeV}$ are assumed. 


\section{Photon-jet production in double Pomeron exchanges processes and Pomeron structure in quarks}

Fig. 3 displays possible observables at the LHC that can probe the quark content in the Pomeron. Fig. 3 (top) shows the $\gamma$-jet to dijet cross section ratio as a function of the leading jet $\mathrm{p}_{T}$ for different assumptions on the quark content of the Pomeron, $\mathrm{d} / \mathrm{u}$ varying between 0.25 and 4 . We notice that the cross section ratio varies by a factor 2.5 for different values of $d / u$ and the ratio depends only weakly on the jet $\mathrm{p}_{T}$ except at low $\mathrm{p}_{T}$, which is due to the fact that we select always the jet with the highest $\mathrm{p}_{T}$ for dijet production (which is obviously different for the $\gamma$-jet sample where we have only one jet at the final state most of the time). The aim of the jet $\mathrm{p}_{T}$ distribution measurement is twofolds: is the Pomeron universal between HERA and the LHC and what is the quark content of the Pomeron? The QCD diffractive fits performed at HERA assumed that $u=d=s=\bar{u}=\bar{d}=\bar{s}$, since data were not sensitive to the difference between the different quark component in the Pomeron. The LHC data will allow us to determine for instance which value of $d / u$ is favoured by data. Let us assume that $d / u=0.25$ is favoured. If this is the case, it will be required to go back to the HERA QCD diffractive fits and check if the fit results at HERA can be modified to take into account this assumption. If the fits to HERA data lead to a large $\chi^{2}$, it would indicate that the Pomeron is not the same object at HERA and the LHC. On the other hand, if the HERA fits work under this new assumption, the quark content in the Pomeron will be further constrained. The advantage of measuring the cross section ratio as a function of jet $\mathrm{p}_{T}$ is that most of the systematic uncertainties due to the determination of the jet energy scale will cancel. This is however not the case for the jet energy resolution since the jet $\mathrm{p}_{T}$ distributions are different for $\gamma+$ jet and dijet events.

Fig. 3 (bottom) displays the $\gamma+$ jet to dijet cross section ratio as a function of the diffractive mass $\mathrm{M}$ computed from the proton missing momentum $\xi$ measured in the forward detectors, $M=\sqrt{\xi_{1} \xi_{2} s}$ where $\xi_{1}$ and $\xi_{2}$ are the momentum fractions of the proton carried by each Pomeron and measured in the proton detectors. The advantage of this variable is that most of systematic uncertainties due to the measurement of the diffractive mass cancel since the mass distributions for $\gamma+$ jet and dijet are similar. The typical resolution on mass is in addition very good of the order of 2 to $3 \%$. The statistical uncertainties corresponding to $300 \mathrm{pb}^{-1}$ (three weeks of data taking at low pile up at the LHC) are also shown on the Fig. 4. This measurement will be fundamental to constrain in the most precise way the Pomeron structure in terms of quark densities, and to test the Pomeron universality between the Tevatron and the LHC. Let us notice that the measurement can still be performed with $100 \mathrm{pb}^{-1}$ (about one week of data taking), but this would increase the statistical uncertainties in Fig. 4 of about $40 \%$. It would still be possible to distinguish between extreme models. $300 \mathrm{pb}^{-1}$ is the optimal luminosity for this measurement in order to get a more precise measurement. Working at higher pile up will require new strategies to be developed, by using for instance fast timing detectors allowing us to measure the proton time of flight which can be used to determine if the protons originate from the main hard interaction or from pile up. 


\section{Comparison with soft color interaction models}

Soft color interaction models (SCI) [5] describe additional interactions between colored partons below the conventional cutoff for perturbative QCD in order to explain the global color singlet exchange. These are based on the assumption of factorization between the conventional perturbative event and the additional non-perturbative soft interactions. Soft exchanges imply that the changes in momenta due to the additional exchanges are very small, whereas the change in the event's color topology due to exchanges of color charge can lead to significant observables, e.g. rapidity gaps and leading beam remnants. The probability to obtain a leading proton at the LHC in the context of SCI models depends on the color charge and the kinematic variables of the beam remnant before hadronization. We find an overall good agreement between Herwig/DPE [6][7] and Pythia/SCI [8] for the prediction of the ratio between $\gamma$-jet and dijet final state cross sections, but the distribution of this ratio as a function of the total diffractive mass distributions may allow to distinguish between the Herwig/DPE and Pythia/SCI models because the latter leads to a flatter dependence to the total diffractive mass, as shown in Fig. 3 (bottom).
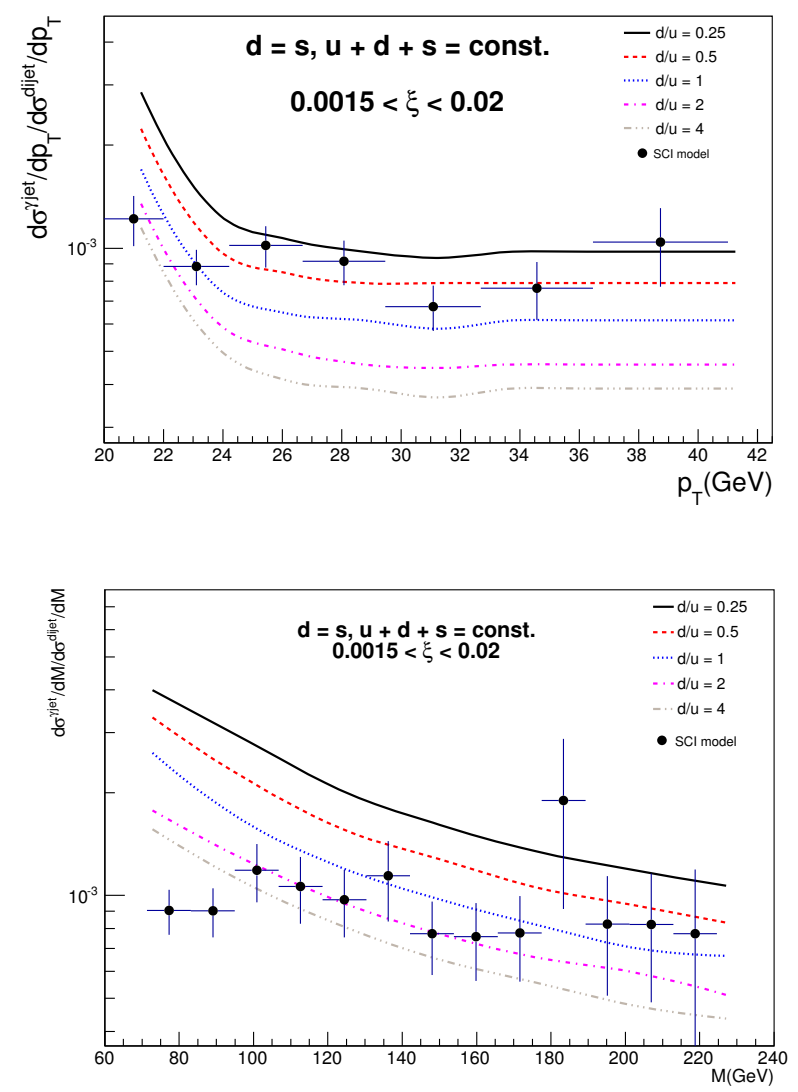

Figure 3: Ratio of $\gamma+$ jet over dijet differential cross section of the leading jet $p_{T}$ (top). Ratio of $\gamma+$ jet over dijet differential cross section as a function of the diffractive mass $M=\sqrt{\xi_{1} \xi_{2} s}$ (bottom). The different curves correspond to different values of $\mathrm{d} / \mathrm{u}$ inside the Pomeron (see text). Proton-proton collisions at $\sqrt{s}=$ $14 \mathrm{TeV}$ are assumed. 

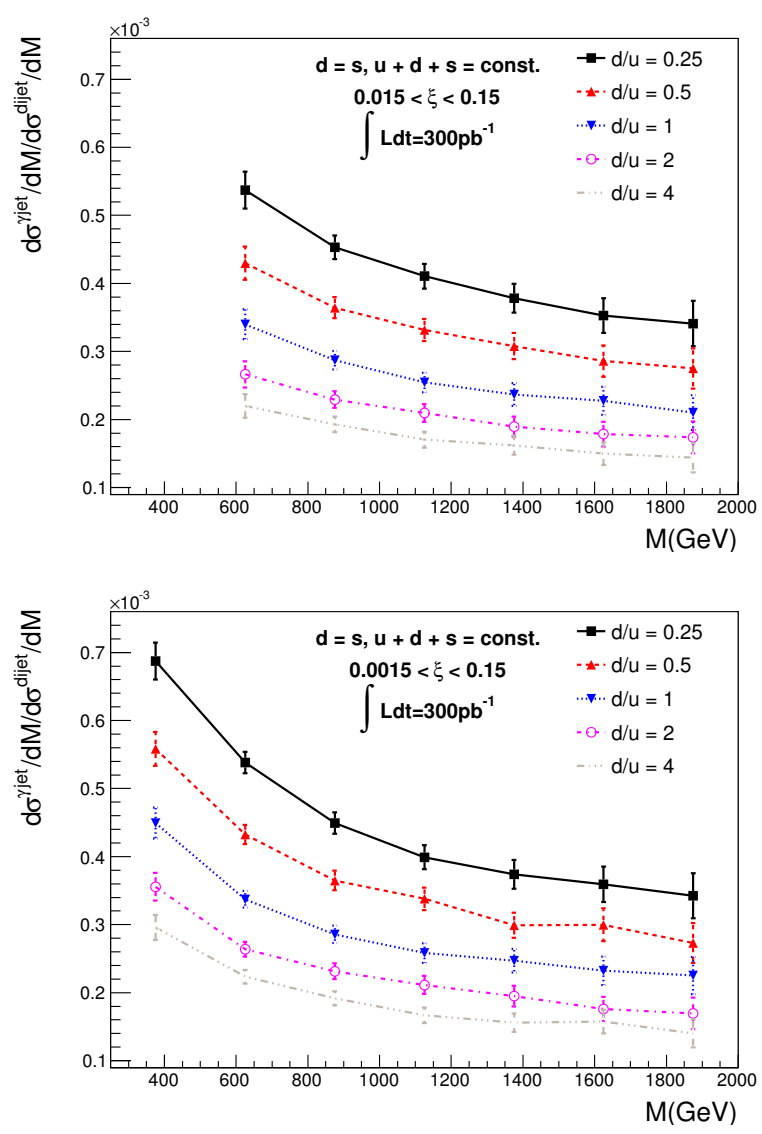

Figure 4: DPE $\gamma+$ jet to dijet differential cross section ratio as a function of the diffractive mass $M=\sqrt{\xi_{1} \xi_{2} s}$ for different values of $\mathrm{d} / \mathrm{u}$ within the acceptance of the $210 \mathrm{~m}$ proton detectors (top) and within the acceptance of both the 210 and $420 \mathrm{~m}$ detectors (bottom).

\section{References}

[1] C. Marquet, C. Royon, M. Saimpert and D. Werder, Phys. Rev. D 88, 074029 (2013).

[2] ATLAS Collaboration, Letter of Intent for the Phase-I Upgrade of the ATLAS Experiment, CERN-LHCC-2011-012.

[3] F. D. Aaron et al., Eur. Phys. J. C 71, 1578 (2011). F. D. Aaron et al. [H1 Collaboration], Eur. Phys. J. C 72, 2074 (2012). S. Chekanov et al. [ZEUS Collaboration], Nucl. Phys. B 800, 1 (2008).

S. Chekanov et al. [ZEUS Collaboration], Nucl. Phys. B 816, 1 (2009). A. Aktas et al. [H1 Collaboration], Eur. Phys. J. C 48, 715 (2006).

[4] O. Kepka, C. Royon, Phys.Rev. D76 034012 (2007).

[5] A. Edin, G. Ingelman and J. Rathsman, Phys. Lett. B 366, 371 (1996); Z.Phys. C75, 57 (1997).

[6] G. Corcella et al., JHEP 0101, 010 (2001).

[7] M. Boonekamp, A. Dechambre, V. Juranek, O. Kepka, M. Rangel, C. Royon and R. Staszewski, arXiv:1102.2531 [hep-ph].

[8] T. Sjostrand, S. Mrenna and P. Z. Skands, JHEP 0605 (2006) 026 [hep-ph/0603175]. 